\title{
CONVOLUTIONS OF CONTINUOUS MEASURES AND SETS OF NONSYNTHESIS
}

\author{
SADAHIRO SAEKI
}

\begin{abstract}
Let $G$ be a nondiscrete LCA group, and $A(G)$ the Fourier algebra of $G$. It is shown that if $E$ is a closed subset of $G$ such that $(\mu * \nu)(E) \neq 0$ for some $\mu$ and $\nu \in M_{c}(G)$, then $E$ is a set of analyticity and contains a set of nonsynthesis for $A(G)$.
\end{abstract}

Salinger and Varopoulos [5] give a condition for a closed subset of a metrizable LCA group to be a set of analyticity. In this note we shall prove that every set satisfying their condition contains a set of nonsynthesis. This will be done by first improving one of their results in [5] and then applying a theorem of Varopoulos [6]. We shall also give improvements of the main results of Rago [3] and Graham [1].

Let $G$ be a nondiscrete LCA group, and $A(G)=\left(L^{1}(\hat{G})\right)^{\wedge}$ the Fourier algebra of $G$. A closed subset $E$ of $G$ is called a set of synthesis if there exists only one closed ideal $I$ in $A(G)$ such that $\cap\left\{f^{-1}(0): f \in I\right\}=E$. If every function defined on the interval $[-1,1]$ that operates in $A(E)=\left.A(G)\right|_{E}$ can be extended to an analytic function on some neighborhood of $[-1,1]$ in the complex plane, then $E$ is called a set of analyticity. Finally we let $M(G)$ denote the convolution measure algebra of $G$, and $M_{c}(G)$ the ideal of all continuous measures in $M(G)$.

THEOREM 1 (CF. [5]). If $E$ is a closed subset of $G$ such that $(\mu * \nu)(E)>0$ for some $\mu$ and $\nu \in M_{c}^{+}(G)$, then $E$ is a set of analyticity and contains a set of nonsynthesis.

To prove this, we need the following improvement of Proposition 1 of [5]. Our proof is notationally complicated, but is an easy consequence of repeated applications of the Fubini theorem.

Proposition 1. Let $X$ and $Y$ be two locally compact (Hausdorff) spaces, and $E$ a Borel subset of the product space $X \times Y$. Suppose that $(\mu \times \nu)(E)>0$ for some $\mu \in M_{c}^{+}(X)$ and $\nu \in M_{c}^{+}(Y)$. Then there exist compact (nonempty) perfect sets $K \subset X$ and $L \subset Y$ such that $K \times L \subset E$.

Proof. By induction on $n \geqslant 1$, we construct a collection $\left\{A_{n j}: 1 \leqslant j \leqslant 2^{n}\right\}$ of disjoint compact subsets of $X$ and also a collection $\left\{B_{n k}: 1 \leqslant k \leqslant 2^{n}\right\}$ of disjoint compact subsets of $Y$. When they are defined, we write as follows:

Received by the editors January 12, 1976.

AMS (MOS) subject classifications (1970). Primary 43A45.

Key words and phrases. LCA group, Fourier algebra, set of analyticity, set of synthesis, independent set, continuous measure. 


$$
\begin{aligned}
r(n) & =2^{n}, \quad E(x)=\{y \in Y:(x, y) \in E\} \quad \text { for } x \in X, \\
A(n) & =A_{n 1} \times A_{n 2} \times \cdots \times A_{n r(n)} \subset X^{r(n)}, \\
\mu_{n j} & =\mu \mid A_{n j} \in M\left(A_{n j}\right) \quad \text { for } 1 \leqslant j \leqslant 2^{n}, \\
\mu_{n} & =\mu_{n 1} \times \mu_{n 2} \times \cdots \times \mu_{n r(n)},
\end{aligned}
$$

and similarly for $B(n) \subset Y^{r(n)}, \nu_{n k} \in M\left(B_{n k}\right), \nu_{n} \in M(B(n))$. We demand that they satisfy

$(\mathrm{a})_{n}$

$$
\int_{A(n)}\left\{\prod_{k=1}^{r(n)} \nu_{n k}\left[\bigcap_{j=1}^{r(n)} E\left(x_{j}\right)\right]\right\} d \mu_{n}(x)>0 .
$$

Without loss of generality, we may assume that $E$ is compact. Write $A(0)=A_{01}=X, \mu_{0}=\mu_{01}=\mu, B(0)=B_{01}=Y, \nu_{0}=\nu_{01}=\nu$, and note that

$$
\int_{A(0)} \nu_{01}(E(x)) d \mu_{0}(x)=(\mu \times \nu)(E)>0
$$

by the Fubini theorem and the hypothesis. Suppose that $\left\{A_{n j}\right\}_{j}$ and $\left\{B_{n k}\right\}_{k}$ as above have been defined for some $n \geqslant 0$. In the following arguments, we shall frequently use the Fubini theorem without reference.

Let $I_{E}$ denote the characteristic function of $E$. For $x=\left(x_{1}, \ldots, x_{r(n)}\right) \in$ $A(n), y=\left(y_{1}, \ldots, y_{r(n)}\right)$ and $y^{\prime}=\left(y_{1}^{\prime}, \ldots, y_{r(n)}^{\prime}\right) \in B(n)$, set

$$
f\left(x, y, y^{\prime}\right)=\prod_{j=1}^{r(n)} \prod_{k=1}^{r(n)} I_{E}\left(x_{j}, y_{k}\right) \cdot I_{E}\left(x_{j}, y_{k}^{\prime}\right)
$$

$z=\left(y, y^{\prime}\right)$, and

$$
g(z)=g\left(y, y^{\prime}\right)=\int_{A(n)} f\left(x, y, y^{\prime}\right) d \mu_{n}(x)
$$

Then we have

$$
\int_{B(n)^{2}} g d\left(\nu_{n} \times \nu_{n}\right)=\int_{A(n)}\left\{\prod_{k=1}^{r(n)} \nu_{n k}\left[\bigcap_{j=1}^{r(n)} E\left(x_{j}\right)\right]\right\}^{2} d \mu_{n}(x)
$$

which is positive by $(a)_{n}$. Therefore the integral of the function

$$
F\left(x, x^{\prime}, x^{\prime \prime}\right)=\int_{B(n)^{2}} f(x, z) \cdot f\left(x^{\prime}, z\right) \cdot f\left(x^{\prime \prime}, z\right) d\left(\nu_{n} \times \nu_{n}\right)(z)
$$

over the set $A(n)^{3}$ with respect to the measure $\mu_{n} \times \mu_{n} \times \mu_{n}$ is also positive by (2). It follows that there exists a point $x^{\prime \prime}=\left(x_{n 1}, x_{n 2}, \ldots, x_{n r(n)}\right) \in A(n)$ such that

$$
\int_{A(n)^{2}} F\left(x, x^{\prime}, x^{\prime \prime}\right) d\left(\mu_{n} \times \mu_{n}\right)\left(x, x^{\prime}\right)>0 .
$$

Now we define 


$$
\begin{aligned}
B_{n k}^{\prime} & =B_{n k} \cap\left[\bigcap_{j=1}^{r(n)} E\left(x_{n j}\right)\right], \quad \nu_{n k}^{\prime}=\nu \mid B_{n k}^{\prime}, \\
B(n)^{\prime} & =B_{n 1}^{\prime} \times B_{n 2}^{\prime} \times \cdots \times B_{n r(n)}^{\prime} \subset B(n), \\
\nu_{n}^{\prime} & =\nu_{n 1}^{\prime} \times \nu_{n 2}^{\prime} \times \cdots \times \nu_{n r(n)}^{\prime}=\nu_{n} \mid B(n)^{\prime} .
\end{aligned}
$$

Then, by (1) and (3), (4) can be written as

$$
\int_{A(n)^{2}} d\left(\mu_{n} \times \mu_{n}\right)\left(x, x^{\prime}\right) \int_{B(n)^{2}} f(x, z) \cdot f\left(x^{\prime}, z\right) d\left(\nu_{n}^{\prime} \times v_{n}^{\prime}\right)(z)>0 .
$$

Since $\mu$ is a continuous measure, the set of all $\left(x, x^{\prime}\right) \in A(n)^{2}$ such that $x_{j}=x_{j}^{\prime}$ for some $1 \leqslant j \leqslant r(n)$ has zero $\left(\mu_{n} \times \mu_{n}\right)$-measure, and a similar assertion holds for $B(n)^{2}$ and $v_{n}^{\prime} \times v_{n}^{\prime}$. It follows from (5) that there exist disjoint compact subsets $A_{(n+1)(2 j-1)}$ and $A_{(n+1) 2 j}$ of $A_{n j}(1 \leqslant j \leqslant r(n))$, and also disjoint compact subsets $B_{(n+1)(2 k-1)}$ and $B_{(n+1) 2 k}$ of $B_{n k}^{\prime}(1 \leqslant k \leqslant r(n))$ for which (a) $)_{n+1}$ holds. (Notice that the integral in (5) with $A(n)^{2}$ and $B(n)^{2}$ replaced by $\left(\prod_{j=1}^{r(n)} A_{(n+1)(2 j-1)}\right) \times\left(\prod_{j=1}^{r(n)} A_{(n+1) 2 j}\right)$ and $\left(\prod_{k=1}^{r(n)} B_{(n+1)(2 k-1)}\right) \times$ $\left(\prod_{k=1}^{r(n)} B_{(n+1) 2 k}\right)$, respectively, is the same as the integral in $(a)_{n+1}$. $)$ This completes the induction.

Now we have by construction:

$$
\begin{gathered}
x_{n j} \in A_{n j} \quad\left(1 \leqslant j \leqslant 2^{n}\right), \\
\left\{x_{n j}\right\}_{j} \times\left(\cup\left\{B_{(n+1) k}: 1 \leqslant k \leqslant 2^{n+1}\right\}\right) \subset E, \\
A_{(n+1)(2 j-1)} \cup A_{(n+1) 2 j} \subset A_{n j} \quad\left(1 \leqslant j \leqslant 2^{n}\right), \\
B_{(n+1)(2 k-1)} \cup B_{(n+1) 2 k} \subset B_{n k} \quad\left(1 \leqslant k \leqslant 2^{n}\right) .
\end{gathered}
$$

We then claim that there exist compact perfect sets $K \subset X$ and $L \subset B$ such that $K \times L \subset E$, where $B=\cap_{n=1}^{\infty}\left(\cup_{k=1}^{r(n)} B_{n k}\right)$. To confirm this, let $\sigma_{n}$ be the probability measure that assigns mass $2^{-n}$ to each $x_{n j}\left(1 \leqslant j \leqslant 2^{n}\right)$, and let $\sigma$ be an arbitrary weak* cluster point of $\left(\sigma_{n}\right)$ in $M(X)$. Then, by (b) and (d), $\sigma$ is a continuous probability measure, since the compact sets $A_{n j}\left(1 \leqslant j \leqslant 2^{n}\right)$ are pairwise disjoint. Moreover, we have (supp $\sigma) \times B \subset E$, since $\left\{x_{n j}\right\}_{n j} \times B \subset$ $E$ by (c) and since $E$ is compact. Similarly we can construct a continuous probability measure $\tau$ on $B$. Consequently it suffices to define $K=\operatorname{supp} \sigma$ and $L=\operatorname{supp} \tau$, which completes the proof.

The Proof of TheOREM 1 is now easy. Let $E, \mu$, and $\nu$ be as in Theorem 1 . Then we have $(\mu \times \nu)(\tilde{E})=(\mu * \nu)(E)>0$, where $\tilde{E}=\left\{(x, y) \in G^{2}: x+y\right.$ $\in E\}$. It follows from Proposition 1 that there exist compact perfect sets $K$ and $L$ in $G$ such that $K \times L \subset \tilde{E}$, or equivalently, such that $K+L \subset E$. Therefore $E$ is a set of analyticity by Theorem 9.3 .5 of [6], and contains a set of nonsynthesis by Theorem 9.2 .3 of [6]. (The metrizability and compactness of $G$ in Theorem 9.2 .3 of [6] can be easily removed.) This establishes Theorem 1.

For a compact space $K$, we write $\mathbf{C}_{p}(K)=\left\{f \in \mathbf{C}(K): f^{p}=1\right\}$ for $p=$ $2,3, \ldots$, and $\mathbf{C}_{\infty}(K)=\{f \in C(K):|f|=1\}$.

Corollary 1. Suppose, in addition to the hypotheses of Theorem 1, that $G$ is metrizable. Then there exist two independent Cantor sets $K, L$ in $G$, and two 
elements $p, q$ of $\{2,3, \ldots, \infty\}$ having the following properties: (a) the set $K+L+y_{0}$ is contained in $E$ for some $y_{0} \in G$, and (b) given $f \in \mathbf{C}_{p}(K)$, $g \in \mathbf{C}_{q}(L)$, and $\varepsilon>0$, there exists a continuous character $\gamma$ of $G$ such that $|f-\gamma|<\varepsilon$ on $K$ and $|g-\gamma|<\varepsilon$ on $L$.

Proof. This follows from Proposition 1 and an easy modification of the well-known method of constructing perfect Kronecker (or $K_{p}$ ) sets (see [4, 5.2.4]). We omit the details.

Now, let us say that a subset $E$ of $G$ is algebraically scattered if to each nonzero element $y$ of $G p(E)$ there exists a countable subset $X$ of $G$ such that $y \notin G p\left(E \cap X^{c}\right)$. Notice that if $E$ is such a set, then so is the set $\{n x: n \in$ $\mathbf{Z}, x \in E\}$, where $\mathbf{Z}$ denotes the set of all integers. We define $n E=\{0\}$ for $n=0$, and $n E=(n-1) E+E$ for $n \geqslant 1$. The following is an improvement of a result of Rago [3] (see also [2]).

THEOREM 2. Suppose that $E$ is a $\sigma$-compact subset of $G$ which is algebraically scattered, and that $\mu_{j} \in M_{c}^{+}(G)$ for $1 \leqslant j \leqslant n+1$. Then we have

$$
\begin{aligned}
\left(\mu_{1} * \cdots * \mu_{n}\right)[(n E+x) \cap(n E+y)] & =0 & & (x \neq y), \\
\left(\mu_{1} * \cdots * \mu_{n} * \mu_{n+1}\right)(n E+y) & =0 & & (y \in G) .
\end{aligned}
$$

Proof. If (i) holds, then there exist at most countably many $x \in G$ such that $f(x) \neq 0$, where $f(x)=\left(\mu_{1} * \cdots * \mu_{n}\right)(n E+x)$. Therefore the integration of $f(y-x)$ with respect to $d \mu_{n+1}(x)$ yields (ii) since $\mu_{n+1} \in M_{c}(G)$. In other words, (i) implies (ii).

Defining $\mu_{1} * \cdots * \mu_{n}=$ the unit point measure at $0 \in G$ when $n=0$, we note that the result is trivial for $n=0$. So assume that $n$ is a natural number, and that the result holds if $n$ is replaced by $n-1$. Given $\mu_{1}, \ldots, \mu_{n}$ $\in M_{c}^{+}(G)$, let $\mu$ be their convolution. To confirm (i), we may assume that $x=0 \neq y$ (if necessary, translate $\mu_{1}$ by $x$ ), and that $n E \cap(n E+y) \neq \varnothing$. Then notice that $y$ is in $n E-n E \subset G p(E)$. Since $E$ is algebraically scattered, there exists a countable set $X$ in $G$ such that $y \notin G p\left(E \cap X^{c}\right)$. Then we have $\left[n\left(E \cap X^{c}\right)\right] \cap\left[n\left(E \cap X^{c}\right)+y\right]=\varnothing$, so that $n E \cap(n E+y)$ is contained in the union of $(n-1) E+X$ and $(n-1) E+X+y$. Since $X$ is countable, the last two sets have zero $\mu$-measure by the inductive hypothesis. Hence $n E \cap(n E+y)$ itself has zero $\mu$-measure, which completes the proof.

Let $E$ be a subset of $G, p \in \mathbf{Z}$, and $n$ a natural number. We define $p \times E=\{p x: x \in E\}, E(0)=\{0\}$, and $E(n)=\cup\left(p_{1} \times E+\cdots+p_{n} \times\right.$ $E)$, where the union is taken over all $\left(p_{1}, \ldots, p_{n}\right) \in \mathbf{Z}^{n}$. Notice that if $K \subset G$ is a compact set with positive Haar measure, then there exists an absolutely continuous measure $\mu \in M^{+}(G)$ such that $\mu^{n}(K)>0$ for all $n \geqslant 1$. Thus the following theorem is a generalization of a result of Graham [1].

THEOREM 3. Suppose that $E \subset G$ is an independent set, that $F \subset G$ is an arbitrary set with Card $F<$ the cardinality of the continuum, and that $\mu_{1}, \ldots, \mu_{n}, \mu_{n+1} \in M_{c}^{+}(G)$. Then we have $\left(\mu_{1} * \cdots * \mu_{n} * \mu_{n+1}\right)(K)=0$ for every compact set $K$ in $F+E(n)$.

To prove this, we need the following.

Proposition 2. Suppose that $E \subset G$ is an independent set, and that 
$X_{1}, \ldots, X_{n}, X_{n+1} \subset G$ are uncountable sets. Then $\sum_{j=1}^{n+1} X_{j} \not \subset F+E(n)$ for any set $F \subset G$ with $C a r d F<\min \left\{\operatorname{Card} X_{j}: 1 \leqslant j \leqslant n+1\right\}$.

Proof. Suppose by way of contradiction that this is false for some $F$ as above:

$$
X_{1}+\cdots+X_{n}+X_{n+1} \subset F+E(n) .
$$

Without loss of generality, we may assume that $n$ is the least one of all such natural numbers, and that every $X_{j}$ contains $0 \in G$. Notice that the latter assumption implies $\sum_{j \neq k} X_{j} \subset \sum_{j=1}^{n+1} X_{j}$ for all $1 \leqslant k \leqslant n+1$, and that (1) cannot happen if $n=0$.

Let $c^{\prime}=\min \left\{\right.$ Card $\left.X_{j}: 1 \leqslant j \leqslant n+1\right\}$, and let $c$ be the least ordinal such that the set $A=\{1,2, \ldots, a, a+1, \ldots\}$ of all ordinals less than $c$ has cardinal equal to $c^{\prime}$. Let also $B$ be the product space $A \times\{1,2, \ldots, n+1\}$ with the lexicographic order. We choose an element $w(a, j) \in \Sigma_{i \neq j} X_{i}$ for each $(a, j) \in B$ as follows. For $(a, j)=(1,1), w(1,1)$ may be an arbitrary element of $\sum_{i \neq 1} X_{i}$. Suppose that the elements $w(a, j) \in \sum_{i \neq j} X_{i},(a, j)<$ $(b, k)$, have been defined for some $(b, k) \in B$. Then we can write by (1)

$$
w(a, j)=y(a, j)+\sum_{i=1}^{n} p_{i}(a, j) x_{i}(a, j),
$$

where $y(a, j) \in F, p_{i}(a, j) \in \mathbf{Z}$, and $x_{i}(a, j) \in E$ for all $(a, j)<(b, k)$ and $1 \leqslant i \leqslant n$. Let $F(b, k)$ denote $G p\left(\left\{x_{i}(a, j): 1 \leqslant i \leqslant n,(a, j)<(b, k)\right\}\right)$, and notice that $\operatorname{Card}(F+F(b, k))<c^{\prime}$, since $c^{\prime}$ is an uncountable cardinal larger than Card $F$. It follows from the minimality of $n$ that the set $\sum_{i \neq k} X_{i}$ contains an element $w(b, k)$ such that

$$
w(b, k) \notin F+F(b, k)+E(n-1) \text {. }
$$

This completes our transfinite induction.

Now (3) implies that the elements $x_{i}(a, j)(1 \leqslant i \leqslant n, 1<a<c, 1 \leqslant j \leqslant$ $n+1)$ are different and that

$$
p_{i}(a, j) x_{i}(a, j) \neq 0
$$

for all $1 \leqslant i \leqslant n, 1<a<c$, and $1 \leqslant j \leqslant n+1$. Moreover, there exist $y_{j} \in F(1 \leqslant j \leqslant n+1)$ such that the set $A_{j}=\left\{a \in A: a>1, y(a, j)=y_{j}\right\}$ has infinite cardinal larger than Card $F$, because Card $A=c^{\prime}>$ Card $F$. Then $a_{1} \in A_{1}, \ldots, a_{n+1} \in A_{n+1}$ imply

$$
\sum_{j=1}^{n+1}\left\{y_{j}+\sum_{i=1}^{n} p_{i}\left(a_{j}, j\right) x_{i}\left(a_{j}, j\right)\right\}=\sum_{j=1}^{n+1} w\left(a_{j}, j\right)
$$

by (2), and the sum in the right-hand side of (5) belongs to the set $\sum_{j=1}^{n+1} \sum_{i \neq j} X_{i}$ $=n\left(\sum_{j=1}^{n+1} X_{j}\right)$. Since the last set is contained in $n(F+E(n))=n F+E\left(n^{2}\right)$ by (1), it follows that there exists $y=y\left(a_{1}, \ldots, a_{n+1}\right) \in n F$ such that

$$
y_{0}+\sum_{j=1}^{n+1} \sum_{i=1}^{n} p_{i}\left(a_{j}, j\right) x_{i}\left(a_{j}, j\right) \in y+E\left(n^{2}\right),
$$

where $y_{0}=\sum_{j=1}^{n+1} y_{j}$. Since each $A_{j}$ has infinite cardinal larger than Card $F$, there must be distinct elements $a_{1}, b_{1}, \ldots, a_{n+1}, b_{n+1}$ in $A$ such that $a_{j}, b_{j} \in$ $A_{j}$ for all $j$ and such that $y\left(a_{1}, \ldots, a_{n+1}\right)=y\left(b_{1}, \ldots, b_{n+1}\right)$. But then we 
have by (6)

$$
\sum_{j}\left\{\sum_{i} p_{i}\left(a_{j}, j\right) x_{i}\left(a_{j}, j\right)-\sum_{i} p_{i}\left(b_{j}, j\right) x_{i}\left(b_{j}, j\right)\right\} \in E\left(2 n^{2}\right),
$$

which contradicts the independence of $E$ by (4) (notice that the elements $x_{i}(a, j), a>1$, are distinct).

To prove Theorem 3, we need the fact that Proposition 1 holds for more than two factors: if $X_{1}, \ldots, X_{n}$ are $n$ locally compact spaces, and if $E$ is a Borel subset of their product space such that $\left(\mu_{1} \times \cdots \times \mu_{n}\right)(E)>0$ for some $\mu_{1} \in M_{c}^{+}\left(X_{1}\right), \ldots, \mu_{n} \in M_{c}^{+}\left(X_{n}\right)$, then there exist compact perfect sets $K_{j} \subset X_{j}(1 \leqslant j \leqslant n)$ such that $K_{1} \times \cdots \times K_{n} \subset E$. This can be proved along the same lines as Proposition 1. Therefore Theorem 3 follows from Proposition 2 and the well-known fact that every compact perfect set has cardinal larger than or equal to the cardinality of the continuum. We omit the details.

\section{REFERENCES}

1. C. C. Graham, Compact independent sets and Haar measure, Proc. Amer. Math. Soc. 36 (1972), 578-582. MR 47 \#2001.

2. S. Hartman and C. Ryll-Nardzewski, Quelques résultats et problemes en algèbre des mesures continues, Colloq. Math. 22 (1971), 271-277. MR 44 \# 1993.

3. J. M. Rago, Convolutions of continuous measures and sums of an independent set, Proc. Amer. Math. Soc. 44 (1974), 123-128. MR 48 \#9258.

4. W. Rudin, Fourier analysis on groups, Interscience Tracts in Pure and Appl. Math., no. 12, Interscience, New York, 1962. MR 27 \#2808.

5. D. L. Salinger and N. Th. Varopoulos, Convolutions of measures and sets of analyticity, Math. Scand. 25 (1969), 5-18. MR 41 \#8930.

6. N. Th. Varopoulos, Tensor algebras and harmonic analysis, Acta Math. 119 (1967), 51-112. MR 39 \# 1911.

Department of Mathematics, Tokyo Metropolitan University, Setagaya, Tokyo, Japan 